\title{
THE FEASIBILITY OF POSTURE AND MOVEMENT DETECTION BY ACCELEROMETRY
}

\author{
Peter H. Veltink ${ }^{1}$, Hans B.J. Bussmann'ㄹ, Frank Koelma ${ }^{1}$, Henry M. Franken ${ }^{1}$, \\ Wim L.J. Martens ${ }^{3}$ and Rob C. van Lummel $^{4}$ \\ 1 Biomedical Technological Institute, University of Twente, \\ P.O. Box 217, 7500 AE Enschede, the Netherlands \\ ${ }^{2}$ Erasmus University, Department of Rehabilitation Medicine, Rotterdam, the Netherlands \\ ${ }^{3}$ PhyVision bv, Gemert, the Netherlands \\ ${ }^{4}$ McRoberts bv, the Hague, the Netherlands
}

\begin{abstract}
The discrimination of postures and movements using a minimal set of uniaxial accelerometers was investigated. Postures and movements were distinguished on the basis of the high-pass filtered, rectified and low pass filtered signal of one accelerometer. Postures were discriminated by combining the constant valued signals of the accelerometers, mounted on different segments of the body. One sensor mounted radially on the trunc and one mounted radially or tangentially on the upper leg appeared to be sufficient to discriminate standing, sitting . and lying. Methods are proposed for the discrimination of different cyclical movements.
\end{abstract}

\section{INTRODUCTION}

In order to optimize and evaluate the rehabilitation treatment of patients it is important to evaluate their daily life activities in their domestic environment. Such an evaluation requires a portable measurement unit and kinematic sensors which do not influence the activities.

In this study, the feasibility of a posture and movement detection scheme using a limited number of uni-axial accelerometers is invesiigated.

\section{DISTINGUISHING POSTURES AND MOVEMENTS USING ACCELEROMETERS}

\section{Accelerometers}

Small uni-axial accelerometers exist which can be easily mounted on the skin. If mounted on a segment of a human body, they measure a component of the actual acceleration of the segment at the site of the sensor, and a component of the equivalent gravitational acceleration, which depends on the orientation of the segment. It is important to use accelerometers which measure also dc-components, like the piezoresistive types. Willemsen et al. proposed methods for the application of these sensors in the analysis of body movement [1].

\section{Distinguishing between postures and movements}

If the sensor signal is not changing over time, it can be assumed that the body segment is not moving, because a constant acceleration or an exact cancelation of acceleration and gravitational components during movements is not to be expected for prolonged periods of time during normal body movements. This characteristic can be used to discriminate between postures and movements: a movement detector was constructed by applying a threshold to the high-pass filtered, rectified and low-pass filtered signal of one of the accelerometers.

\section{Discriminating postures}

If no movement is detected, it is assumed that the body is in a posture. By combining the value of several accelerometers mounted on different body segments several postures can be discriminated. The postures standing, sitting and lying can be distinguished by the orientations of only the trunc and one upper leg. Therefore, two accelerometers with their sensitive axes in the sagittal plane are sufficient to discriminate between these postures: one accelerometer radially mounted on the trunc and one radially or tangentially mounted on an upper leg. A tangentially mounted trunc accelerometer can not be used, because lying on a side would not be distinguishable from standing. Combining a radially mounted trunc accelerometer and a tangentially mounted upper leg accelerometer also enables the discrimination of lying on a side. lying on the back and lying face down. If more postures or more aspects of postures have to be distinguished, more sensors might be required.

\section{Discrimimating cyclical movements}

Directly after a movement has been detected, the cyclical nature of the movement can be investigated. Potentially applicable time domain analysis methods include the detection of repeating peaks in the signals. Combined time and frequency domain methods include locking in onto the cycle frequency by instantaneous frequency analysis [2], tracing 
variations in cycle frequency while walking at varying speeds.

Potential methods for the discrimination of cyclical movements include multi-correlation analysis (simultaneously applied to all accelerometer signals) and combining the average signal values per cycle for all sensors.

\section{EXPERIMENTAL METHODS}

Experiments were performed on five healthy subjects, who performed several activities, including the postures and movements of interest, according to a strict and supervised protocol. Up to 6 uni-axial accelerometers (IC sensors ${ }^{\mathrm{TM}}, 5 \mathrm{~g}$ ) were simultaneously. mounted on the trunc, upper legs and lower legs in varying orientations. Footswitches were placed under the feet and a marker switch was operated by the subject to mark the beginning and end of activities. The sensor signals were sampled and stored on a portable measurement system (Vitaport ${ }^{\mathrm{TM}}$ ).
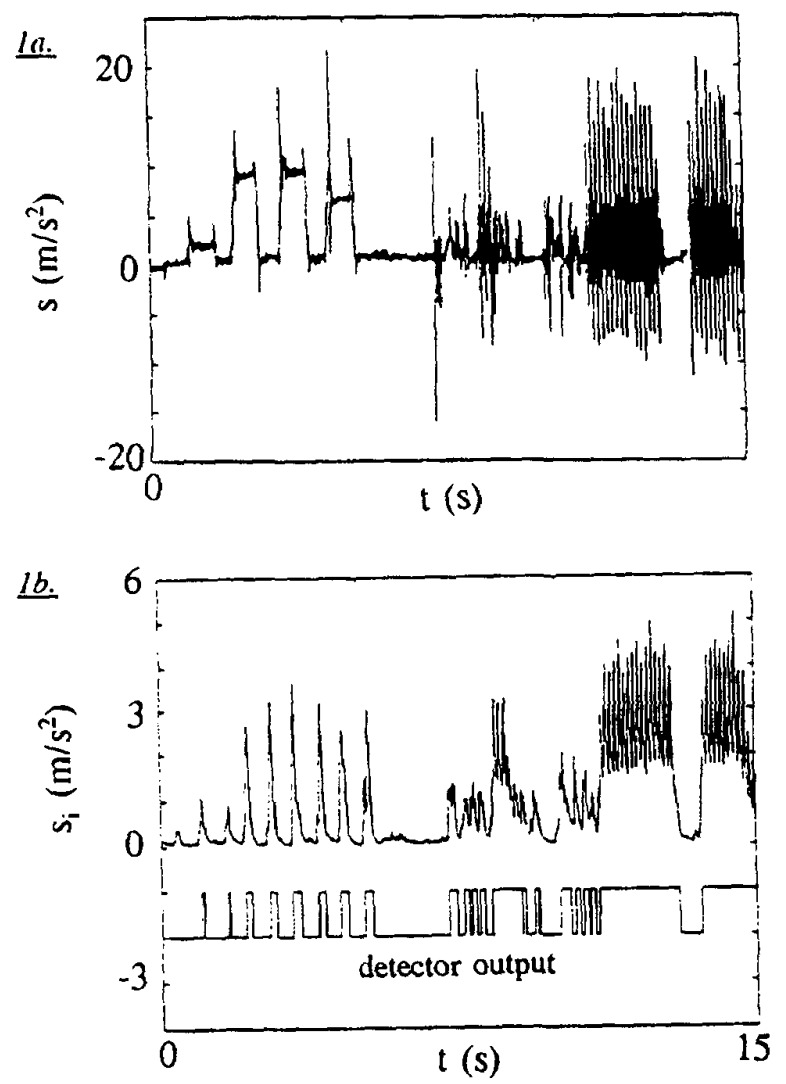

Figure 1. Typical performance of the movement detector. a. Signal of tangentially mounted upper leg accelerometer. b. The same signal afier high-pass filtering first order Butterworth filter, $f_{c}=0.5 \mathrm{~Hz}$ ), rectification and low-pass filtering (first order Butterworth filter, $f_{\mathrm{g}}=0.5 \mathrm{~Hz}$ ), and the movement detector output (threshold $0.5 \mathrm{~m} / \mathrm{s}$ ).

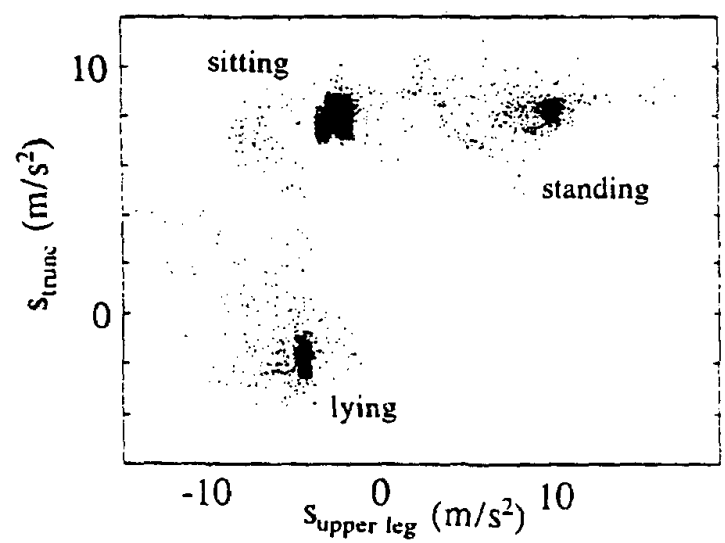

Figure 2. discriminating the postures standing, sitting and lving using one accelerometer radially mounted on the upper leg (x-axis) and one accelerometer radially mounted on the trunc (y-axis).

\section{RESULTS}

Postures and movements could be distinguished, using first order Butterworth high and low pass filters at cut-off frequencies of $0.5 \mathrm{~Hz}$ and a threshold of $0.5 \mathrm{~m} / \mathrm{s}^{2}$ (figure 1).

The discrimination between standing, sitting and lying using one accelerometer on the trunc and one on the upper leg is illustrated in figure 2.

The discrimination between various movements has not be analyzed sufficiently yet. Simply considering the average values of the accelerometer signals per cycle already appeared to discriminate between some cyclical movements.

\section{DISCUSSION}

This study indicates that, in principle, distinction of postures and movements using dc-accelerometers is possible. However, only a limited number of postures and movements were considered, according to a preset protocol. The applicability of this method for activity monitoring of patients suffering from motor control disorders, moving in their home environment, has still to be investigated.

Accelerometers seem to be better suited for activity measurements than conventional kinematic sensors like goniometers because they have small sizes and weights, are easily mountable and do not cross joints.

\section{REFERENCES}

[1] A.Th.M. Willemsen, J.A. van Alsté and H.B.K. Boom, "Real-time Gait Assessment utilizing a new way of Accelerometry", J. Biomechan., vol. 23, pp. 859-863, 1990.

[2] W.L.J. Martens, "The Fast Time Frequency Transform (F.T.F.T.): a novel on-line Approach to the Instantaneous Spectrum", Proc. IEEE Eng. in Med. \& Biol. Soc., Paris, pp. 2594 - 2595, 1992. 